\title{
NOCIONES Y PRINCIPIOS DE LA EDUCACIÓN INTERCULTURAL PRESENTES EN PRÁCTICAS PEDAGÓGICAS REALIZADAS EN CONTEXTO DE INTERACCIÓN JUVENIL RURAL DE LA REGIÓN METROPOLITANA (CHILE)
}

\author{
CONCEPTS AND PRINCIPLES OF THE PRESENT INTERCULTURAL \\ EDUCATION IN TEACHING PRACTICES MADE IN CONTEXT OF RURAL \\ YOUTH INTERACTION METROPOLITAN REGION (CHILE)
}

\author{
Carol Morales Trejos*
}

\begin{abstract}
Este artículo muestra resultados de la investigación "prácticas pedagógicas en contextos de interacción juvenil rural, de la Región Metropolitana de Chile: un análisis desde la educación intercultural" desarrollada en el Doctorado en Ciencias de la Educación mención Educación Intercultural de la Universidad de Santiago de Chile, durante el periodo 2012-2015. Dentro de las finalidades del estudio se encuentran identificar fundamentos teórico y prácticos de la educación intercultural que están presentes en las prácticas pedagógicas de docentes que se desempeñan en contextos de interacción juvenil rural dentro de establecimientos de educación media. Para lo anterior, se realizó una investigación etnometodológica, cuyo enfoque teórico metodológico fue el hermenéutico-interpretativo, empleando entrevistas a profundidad a 14 docentes de seis liceos municipalizados ubicados en seis comunas rurales de la Región Metropolitana de Chile. Los resultados muestran de manera integrada la presencia de nociones y principios del enfoque intercultural, tanto en la caracterización sobre la propia práctica pedagógica como en las representaciones docentes sobre lo juvenil, la diversidad, la cultura, la ruralidad, la educación rural y la interculturalidad. Las conclusiones de esta investigación, permiten considerar que una aproximación a la juventud en contextos rurales desde el enfoque intercultural, permite generar condiciones para el reconocimiento, valoración e incorporación de su cultura a nivel educativo.
\end{abstract}

Palabras claves: educación, prácticas pedagógicas, diversidad, juventudes, ruralidad, interculturalidad.

This paper presents results of the research "Teaching practices in the context of rural youth interaction, of the Metropolitan Region of Chile: An analysis within the intercultural education" developed in the Doctorate of Science Education and a major of Intercultural Education of the University of Santiago, Chile during the period 2012-2015. Among the objectives of the study is to identify theoretical and practical foundations of intercultural education that are present in pedagogical practices of teachers working in rural areas and in youth interaction contexts within secondary education establishments. For this reason, an ethnomethodology research was conducted, with a theoretical approach, using in depth hermeneutic-interpretive interviews with 14 teachers in six municipal high schools located in six rural districts of the metropolitan region of Chile. As a result, it shows in an integrated manner the presence of principles and notions of intercultural approach, both in the characterization of teaching practice and teaching about youth, diversity, culture, rural life, rural education and interculturality. The findings of this research, support the view that an approach to youth in rural settings from the intercultural approach, can create conditions for the recognition, measurement and incorporation of culture to education.

Key words: education, teachers, pedagogical practice, diversity, youth, interculturality.

\section{Introducción}

La formación del profesorado en materia de interculturalidad ha estado fuera de los planteamientos generales de la formación didáctica del docente, tanto en su formación inicial como en los planes de formación permanente (Aguado et al., 2008), asimismo recientes estudios en materia de interculturalidad tanto en América Latina como en Europa realizados en contextos educativos, revelan la necesidad de abrir la formación intercultural a toda la comunidad educativa, espacio en el que resulta ineludible educar en el marco del aprendizaje de competencias interculturales, sin embargo para que se dé este paso a la acción, se requiere en primer instancia realizar un análisis desde la mirada intercultural del actual desarrollo de las prácticas pedagógicas y los principales debates teóricos que se han desarrollado en torno a la temática, como plantea la investigación a la que hace referencia este artículo científico.

\footnotetext{
* Universidad de Costa Rica, Costa Rica. Correo electrónico: carolgraciela.morales@ucr.ac.cr
} 
En este contexto, el objetivo del artículo es adentrarse en identificar fundamentos teóricos y prácticos de la educación intercultural de manera específica en prácticas pedagógicas en contextos de interacción juvenil rural, espacios poco estudiados, cuyo conocimiento facilita elementos para agenciar propuestas de formación y capacitación docente pertinentes al contexto en este caso el chileno, en competencias interculturales o bien pedagogía intercultural.

Los referentes contextuales, empíricos y teóricos en los que se sustentó la investigación citada, ubican a las prácticas pedagógicas, la interculturalidad, la diversidad y la cultura rural en un amplio espacio de debate y posibilidad de generar nuevos conocimientos relevantes (Jurado y Tobasura, 2012; Erazo, 2011; Aguado et al., 2008; Merchán 2001; Cabrera 2009; Kessler, 2005) que favorezcan el desarrollo de una propuesta en educación intercultural en el contexto educativo, centrado en una buena parte en las prácticas docentes, en miras de contribuir en la construcción de una sociedad más equitativa y con una nueva mirada de ciudadanía.

En razón de estas consideraciones es importante tener claridad que la interculturalidad es un enfoque que permite comprender la diversidad, y la cultura como distintas posibilidades de lo diverso cuya premisa es el aprendizaje y la riqueza de saberes, se reafirma dicha importancia por distintos organismos internacionales como la UNESCO y SITEAL, los cuales enfatizan que esta debe ser considerada un eje estructurador del currículum nacional, es decir, como proyecto de Estado, y de forma más precisa una dimensión clave dentro de la práctica pedagógica.

Los debates sobre la diversidad en el contexto educativo, específicamente en cómo esta diversidad es abordada en la interacción pedagógica, ha generado críticas en tanto ha estado asociado a problema o deficiencia, imposibilitando su gestión como una oportunidad de aprendizaje y riqueza, aspecto que constituye un punto medular en este estudio, en tanto se busca facilitar desde el acercamiento de lo juvenil desde la mirada intercultural, nuevas y más integradoras formas de reconocer, valorar e incorporar la diversidad juvenil en espacios educativos rurales.

Por tanto, es clave dentro de las prácticas pedagógicas desde una mirada intercultural, como propone este artículo, que la diversidad se desligue del concepto de problema (Aguado et al., 2006), ya que de lo contrario se promueve una mirada sesgada del otro, para lo cual reflexionar en las creencias respecto del otro en un primer momento (Aguado et al., 2008), (Dietz, 2003) y luego en todo aquello que permita gestionar a ese otro en tanto diverso, constituye un principio de la educación intercultural.

A continuación se presentan las consideraciones metodológicas de la investigación citada, seguida de una breve discusión teórica de los principales referentes que considera este artículo, así como una síntesis de resultados y consideraciones finales del estudio realizado.

\section{Consideraciones metodológicas}

El objetivo general empírico de la investigación de la que se extraen los datos que se muestran en este artículo, consistió en:

Analizar los fundamentos teórico y prácticos de la educación intercultural que están presentes en las prácticas pedagógicas de docentes que se desempeñan en contextos de interacción juvenil rural dentro de establecimientos de educación media en la Región Metropolitana de Chile.

Asimismo en relación con los objetivos específicos, en este artículo se presentan resultados de dos de los cuatro, que dan respuesta al objetivo general anteriormente citado, correspondiente a los siguientes:

1. Determinar que nociones y principios de la educación intercultural están presentes en las representaciones sobre lo juvenil, la diversidad, la cultura, la ruralidad, educación rural e interculturalidad que sustentan las prácticas pedagógicas.

2. Identificar las nociones y principios de la educación intercultural que están presentes en las prácticas pedagógicas de docentes que se desempeñan en contextos de interacción juvenil rural dentro de establecimientos de educación media en la Región Metropolitana de Chile.

La fundamentación epistemológica permite dar cuenta mediante la construcción de un discurso científico hermenéutico el fenómeno que se planteó estudiar, para lo cual se consideraron planteamientos de autores como Vasilachis de Gialdino (2009) y Zemelman (1992) respecto de los fundamentos ontológicos y epistemológicos de la investigación 
cualitativa. Aunado a esta fundamentación epistemológica se contó con una fundamentación contextual, empírica y teórica empleada como insumo en la toma de decisiones metodológicas.

Considerando el método científico hermenéuticointerpretativo dentro del cual esta investigación se posicionó, se planteó un diseño de investigación etnometodológico caracterizado por la flexibilidad y la multiplicidad de escenarios o actores sociales que abarca, ya que corresponde a un estudio que selecciona un número menor de aspectos del fenómeno en estudio aplicado a un número mayor de actores sociales dentro del fenómeno (Erazo, 2012).

Este diseño según Galindo (2009) presupone el reconocimiento de la capacidad reflexiva e interpretativa propia de cada actor social aspecto que constituye el corazón mismo de la etnometodología, ya que la forma de conocimiento práctico es esa facultad que todo individuo posee y aplica en la rutina de sus actividades prácticas. La interpretación se plantea como algo indisociable de la acción, y al mismo tiempo algo compartido por el conjunto de los actores sociales, en este sentido la etnometodología sostiene que en las ciencias sociales todo es interpretación y que "nada habla por sí mismo".

Los escenarios de esta investigación lo configuraron cinco liceos politécnicos y uno científico-humanista de la Región Metropolitana de Santiago de Chile, todos municipalizados, y ubicados en seis comunas consideradas rurales y distribuidas por grupos de ruralidad 1, 2 y 9 . Los sujetos de investigación fueron 14 docentes jefes de los cursos de tercero y cuarto medio, 7 de ellos con formación pedagógica y las 7 personas restantes formación técnica variada. El primer contacto se realizó en la Dirección Regional de Educación de cada municipio, posterior a la autorización se contactó a las personas encargadas, directora o director de los establecimientos educativos, y al contar con el apoyo de la dirección se procedió a buscar de forma individual a docentes jefes de tercero y cuarto medio, quienes de forma voluntaria brindarán sus conocimientos con relación al tema estudiado, resguardando en el proceso las consideraciones éticas pertinentes.

La principal técnica de recogida de información para desarrollar este estudio fueron las entrevistas en profundidad, específicamente se realizaron tres entrevistas en profundidad a cada docente que oscilaron entre los 45 minutos y una hora, cuya pertinencia metodológica radicó en "aprender sobre lo que realmente es importante en la mente de los informantes: sus significados, perspectivas y definiciones; el modo en el que los actores ven la realidad o en la que clasifican y experimentan el mundo..." (Canales, 2006. p. 241).

En torno al procedimiento de análisis y validación de la información recolectada, para los propósitos de la investigación citada, se empleó el análisis de contenido, el que según Delgado y Gutiérrez (2007) es un tipo de análisis cualitativo, que se centra en el análisis de las expresiones verbales tanto orales como escritas.

Cáceres (2003, pp. 58-68) retomando el modelo de desarrollo deductivo-inductivo del análisis de contenido tradicional propuesto por Mayring (2000) y el enfoque del método comparativo constante de Glaser y Strauss (1999), establece una serie de pasos en el procedimiento de análisis de contenido, de los que el estudio doctoral consideró tres de los seis pasos propuestos, asimismo recogió los planteamientos sobre el desarrollo de la técnica de análisis de contenido propuestos por Erazo (2012), que a continuación se detallan.

Primer paso: considero el ajuste de la tabla de consistencia de la investigación, formulación de categorías y subcategorías, y definir las unidades de análisis en este caso frases con sentido completo en un párrafo, siguiendo a Briones (1988b) dichas unidades representaron el contenido significativo dentro del documento que sirvió para extraer resultados.

Segundo paso: en este paso se realizó propiamente la codificación progresiva de todo el corpus del contenido disponible, es decir, se identificaron en el texto las unidades de análisis para cada categoría y subcategoría que posteriormente se introdujeron en tablas descriptivas por docente.

Tercer paso: el tercer paso corresponde al análisis descriptivo, para el que se consideraron tres etapas, la primera de ellas una descripción de primer nivel, seguida de una descripción de segundo nivel, etapas en las que se consideraron las semejanzas, diferencias, tendencias entre los discursos acerca de las categorías y subcategorías, así como los criterios, grupos de ruralidad, origen geográfico y formación pedagógica o técnica empleados en las agrupaciones y comparaciones constantes que se realizaron. La tercera etapa de este paso dio lugar a convertir las columnas de análisis de segundo nivel en texto dentro del capítulo de resultados, 
cuya presentación se estructuró considerando las preguntas de investigación.

Cuarto paso: este correspondió al análisis interpretativo etapa final del proceso, en este momento se integraron los hallazgos en el documento, para la que empleando las ultimas columnas de análisis descriptivo mencionadas anteriormente, se procedió a interpretar teóricamente considerando los objetivos tanto empíricos como teóricos de esta investigación elementos centrales de la construcción teórica final, reflejada tanto en las conclusiones como en las proyecciones de la investigación.

Consideraciones procedimentales que permiten asegurar la validez de la información que se obtiene, en tanto se relacionan con criterios de rigor científico importantes como la confiabilidad, validez, sistematización, consistencia, optimización de los procedimientos, comunicabilidad.

\section{Discusión Teórica}

\section{Práctica pedagógica; conceptualización y aportes desde la Educación Intercultural}

La práctica pedagógica supone una serie de consideraciones teóricas y operativas, dentro de las cuales se puede según lo intercultural como hermenéutica planteada por Abdallah-Pretceille (2006), determinar la presencia de un enfoque intercultural. Desde la mirada intercultural, una práctica pedagógica para que sea considerada intercultural debe ser reflexiva y crítica, siguiendo autores como Rodríguez (2007), Erazo (2001), Aguado et al. (2008), Dietz (2003), quienes además señalan que instalar la reflexión en la práctica pedagógica constituye una significativa oportunidad de aprendizaje.

Esta investigación práctica pedagógica ha sido definida como un conjunto de actividades críticas, reflexivas, contextualizadas y creativas que lleva a cabo la persona profesional en educación para significar, actualizar y recrear tanto los saberes teóricos correspondientes a conocimientos adquiridos en la formación profesional como los saberes de la experiencia individual/colectiva, favoreciendo en la interacción pedagógica la construcción del aprendizaje (Erazo, 2001/2011; Barcena, 2000; Carr y Kemis, 1986; Perrenound, 2007; Suárez, 2001; Villalta, 2009).

Un reto inicial en este sentido lo configura el superar el distanciamiento entre teoría y práctica, ya que por mucho tiempo se ha brindado dentro del perfeccionamiento docente mayor atención a los saberes teóricos, dejando de lado los saberes pedagógicos o experienciales, Suárez (2001) considera que este tema no es ajeno al campo pedagógico, dentro del cual distintos teóricos (Flórez, 1994; Kemmis, 1995; Porlán, 1994) han tratado de explicar dicho distanciamiento, atribuyendo causas como la rigidez en el método positivista en la interpretación de la realidad que instaura una perspectiva unilateral de las cosas, limitando como señala Carr (1995) la comprensión de las circunstancias concretas en que nos encontramos y nuestras ideas sobre lo que es posible.

Empleando el enfoque intercultural como metáfora de la diversidad en educación, implicaría dentro de este proceso reflexivo, siguiendo a Aguado (2011), que la persona educadora no se detenga en el referente cultural como determinante de comportamientos, sino sobre la manera en que la persona emplea los rasgos culturales para decir y decirse, en este sentido es preciso pensar desde la condición de que somos iguales en términos de dignidad, dejando de utilizar la "diversidad" como pretexto para la exclusión social.

En esta línea la comprensión es clave dentro de las prácticas pedagógicas conocer cuáles son las fuerzas que nos alejan de conseguir los mejores logros de nuestros estudiantes, Aguado (2011) considera que en primer lugar están las creencias acerca de las ideas sobre diversidad y la forma de entenderla en el proceso de enseñar, desde un enfoque intercultural el diálogo se hace necesario en esta articulación de teoría y práctica.

Exploración de creencias que dentro de esta investigación consistió en conocer sobre la representación docente acerca de lo juvenil, la diversidad, la cultura, la educación rural y la intercultural, empleando para dicha construcción de representación tres dimensiones, la cognitiva, la afectiva y la conductual referidas por Moscovic citado por Jodelet (1986), entendiendo por representaciones sociales según Bueno-Abad (2000) maneras de interpretar la realidad cotidiana, por medio de la actividad mental llevada a cabo por los individuos y los grupos, cuya finalidad es la de tomar posición respecto de situaciones, hechos, objetos y comunicaciones que les conciernen, asimismo, son "formas de conocimiento socialmente elaboradas y compartidas, es a la vez el proceso de una actividad y la apropiación de la realidad, teniendo una visión práctica y concurrente a la 
construcción de una realidad común en un conjunto social" (p. 31).

Las prácticas pedagógicas desde la mirada de la educación intercultural pretenden contribuir en un nuevo planteamiento de la educación, siguiendo a Torres (2001, p. 229)

(...) el papel del maestro en la educación de los ciudadanos en una sociedad democrática moderna radica en tres aspectos a saber: permitir que los estudiantes afirmen su identidad de género, racial y cultural, proporcionarles la libertad de funcionar más allá de los limites étnicos y culturales y permitirles obtener las aptitudes necesarias para vivir en una sociedad democrática moderna (...).

En este proceso de conocimiento, aprendizaje $\mathrm{y}$ reconocimiento de la identidad se hace valioso reconocer la posibilidad de la inconmensurabilidad de los discursos y la contradicción potencial del propio interés, mediante el desarrollo de canales de diálogo, aspecto último central en las prácticas pedagógicas interculturales, según Sagastizabal y otros (2009) la construcción del diálogo intercultural se debe cimentar en "códigos comunes que nos enriquezcan, reconociendo nuestras diferencias y que nos acerquen reconociendo nuestras similitudes, justamente, la metáfora de la interculturalidad es el diálogo" (p. 76).

Otra especificidad importante la constituye el realizar prácticas pedagógicas en contextos de interacción juvenil rural, en este sentido cabe señalar que la interacción ha sido entendida de acuerdo con Velazco (2007) como la base de la relación simbólica que existe entre uno y otro sujeto en un momento determinado en el que debe existir un sentido de la relación entre ambas, es decir, debe darse un diálogo en el que lo conductual y lo cognitivo confluyan en los individuos.

En esta interacción docente-estudiante, considerando el contexto se hace imprescindible, reconocer que territorio y escuela, no son sistemas estáticos, "si no en movimiento, transformación y reconstrucción permanente a partir de fuerzas exógenas cuya mayor expresión es la globalización y de otras endógenas asociadas a la movilización de los recursos humanos, culturales y naturales de los territorios locales y su capital cultural comunitario" Williamson (2008, p.18), elemento que constituye un referente contextual importante a ser atendido en la interacción pedagógica desde una mirada mutua permanente entre la comunidad educativa, local y global desde la memoria histórica instalada en la referencia respecto del "otro", elemento que configura un gran desafío para la persona profesional en educación que labora en estos contextos de interacción juvenil rural.

Según las consideraciones anteriores, poner el acento dentro de las prácticas pedagógicas, en elementos como el diálogo, el respeto y el rescate del sujeto juvenil en tanto diverso, compartir la diversidad del otro, implican como plantean Dietz (2003) y Aguado et al. (2008) realizar una puesta a disposición del otro de todo aquello que le posibilite ser distinto de lo que es en algún aspecto, para lo que se hace necesario resignificar el espacio educativo, bajo un itinerario plural, crítico y creativo, sin patrones ni reglas rígidas que obstaculicen el trayecto y enfaticen resultados excluyentes (Duschatzky y Skliar, 2000).

El mirar desde lo intercultural involucra, siguiendo a Walsh (2005, p.9), intervenir en las distintas realidades sociales, políticas, económicas, de saberes y conocimientos. Dentro del contexto educativo, la interculturalidad siguiendo a la autora, debe ser "un proceso dinámico y permanente de relación, comunicación y aprendizaje entre culturas en condiciones de respeto, legitimidad, mutua simetría e igualdad" (2005, p.10), con el fin de construir sociedades más justas.

De este modo, los establecimientos educativos se deben constituir como un espacio de reflexión, de debate, de convivencia, de interacción, de adquisición de saberes donde las experiencias vitales y personales, aportadas a este nuevo escenario, cobran relevancia en el intercambio entre los miembros de la comunidad educativa. Se trata, en definitiva, de crear un espacio social regido por nuevas pautas nacidas de la negociación y de la creatividad colectiva, como menciona Betancourt (2004) va más allá de la tolerancia de formas de vida distintas o de formas de escuela distintas, ya que convivir con maneras distintas de vivir y de educar es compartir la diversidad del otro. En la acción pedagógica implicaría reconocer la dimensión mediadora que todo profesional en la educación ejerce para favorecer el respeto y valoración de la diversidad, de forma holística en todas las dimensiones del proceso educativo (Aguado y Herraz, 2006).

De forma puntual es relevante precisar que la interculturalidad es conceptualizada según los 
referentes teóricos y prácticos expuestos como un enfoque educativo basado en el respeto, la equidad, la interacción y el diálogo, que busca favorecer en el proceso educativo bajo un modelo crítico-reflexivo de actuación el reconocimiento, valoración e incorporación de la diversidad sociocultural, promoviendo para tal fin la realización de prácticas pedagógicas interculturales que involucren a los distintos actores educativos (Aguado, 1995; Leiva, 2011; Dietz, 2003. Rehaag, 2010; Escarbajal, 2011), aspectos que se evidencian en el modelo de actuación intercultural propuesto a continuación.

\section{Hacia un modelo en perspectiva intercultural a emplearse en el abordaje de la diversidad en el contexto educativo}

A continuación se describen los tres elementos centrales que configuran el modelo de actuación intercultural denominado; (re) pensando la diversidad (Figura 1), que ha sido enriquecido según planteamientos de diversos autores señalados en este apartado y con los resultados de la investigación, que evidencian la necesidad como menciona Walsh (2005) de comprender la interculturalidad como proceso cognitivo y procedimental de capacidades conceptuales y de habilidades y destrezas específicas, que pueden considerarse competencias interculturales que se desarrollan y fortalecen de manera dialéctica en este modelo.
Las competencias interculturales que se pueden favorecer con la implementación didáctica de este modelo, como eje central en capacitación y formación profesional, se encuentran:

- Capacidad reflexiva y crítica.

- Disposición/sensibilidad hacia la diversidad.

- Desarrollo de habilidades como el diálogo, la escucha, el trabajo colaborativos, la mediación, $\mathrm{y}$ el intercambio.

- Conocimiento de nociones y principios de la educación intercultural.

- Reconocimiento y configuración de la identidad personal en referencia al otro, es decir, como proceso intersubjetivo (proceso de autoconocimiento).

- Habilidad de mediación y negociación intercultural entendiendo la relación entre culturas como dinámico, enriquecedor y dialéctico, asimismo como contradictorio y conflictivo.

- Comprensión de la diversidad y la cultural desde referentes flexibles, variados, dinámicos y cambiantes, no puros y rígidos.

- La habilidad de realizar prácticas pedagógicas Interculturales.

- Aprender a vivir juntos

- Apreciar la diversidad del otro

- Superación de prejuicios/prácticas discriminatorias.

- Comprender la cultura del otro para interactuar, no para acumular conocimiento sobre su cultura.

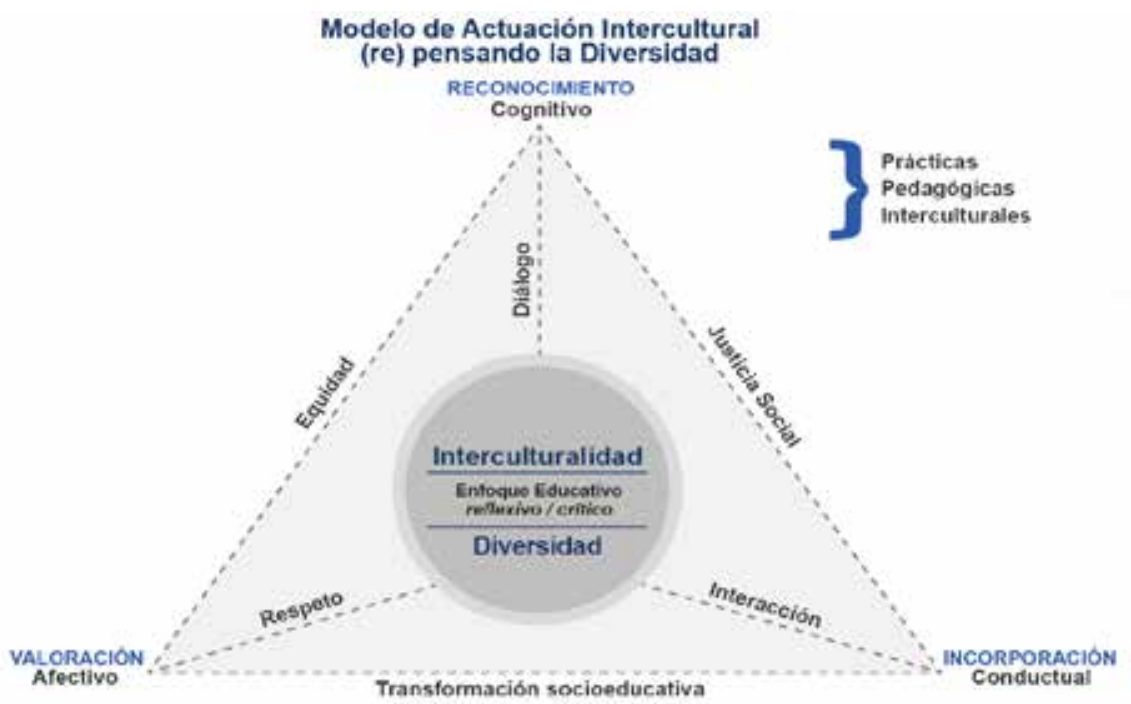

Figura 1. Modelo de Actuación Intercultural.

Fuente: Elaboración propia, considerando; Abdallah-Pretceille, (2006); Aguado y Del Olmo (2009); Leiva, 2011; Dietz, 2003; Rehaag, 2010; Escarbajal, 2011; Walsh, 2005 y datos de la investigación citada. 


\section{Descripción del modelo}

Modelo que recoge nociones y principios de la educación intercultural y los articula operativamente en tres áreas, que se plantean deben ser abordadas en forma conjunta para la implementación de una práctica pedagógica intercultural. Es un modelo cuya aplicación se puede realizar tanto en educación formal como informal, en la formación inicial docente, como en procesos de perfeccionamiento de docentes en ejercicio, y en el caso de la investigación que se cita en este artículo, a nivel teórico ha sido utilizado para mirar las prácticas pedagógicas, identificando nociones y principios interculturales para cada una de estas áreas en las diversas representaciones docentes.

Cabe señalar que es un modelo dinámico, que mantiene sus tres componentes como referentes generales, pero los que se pueden enriquecer adaptándose a las especificidades de la diversidad (étnica, racial, sexual, religiosa, juvenil, cultural, contextual, sociopolítica) que se pretenda comprender y abordar en un contexto determinado, desde planteamientos pedagógicos.

\section{Reconocimiento (cognitivo/reflexión-crítica)}

1. Disposición hacia la reflexión y el cuestionamiento del accionar socioeducativo.

2. Legitimar la existencia del otro, avanzar del conocimiento del otro al reconocimiento, lo que implica revisión de creencias, identificación de estereotipos y hacerse cargo de ellas mediante un proceso de autoconocimiento intersubjetivo.

3. Reconocer la magnitud de lo que implica legitimar al otro, desde la equidad y la justicia social como principios articuladores de la transformación socioeducativa.

4. Disposición a compartir el mundo del otro desde una perspectiva histórica y actual.

5. Reconocer lo propio y lo ajeno, las similitudes y diferencias, identificando puntos de encuentro intercultural que favorezcan la convivencia (pensar en el problema de los encuentros).

\section{Valoración (afectivo/actitudinal)}

1. Considerar que en los referentes sociales, económicos, culturales, religiosos, sexuales y políticos, que definen lo propio y lo ajeno existe una gran riqueza, valoración que permite establecer relaciones simétricas.

2. Reconocer que en la diversidad de seres, saberes y prácticas se pueden generar aprendizajes importantes para seguir avanzando en la construcción de sociedades más justas y equitativas.

3. Valorar la diversidad sociocultural desde sentidos amplios y dinámicos no excluyentes.

\section{Incorporación (conductual/procedimental)}

1. Conciencia de que la interculturalidad en el espacio educativo no es un contenido, sino un proceso vivencial de trabajo social y político, basado en la interacción entre personas, conocimientos y prácticas culturalmente diferentes.

2. Reconocer en la interacción cotidiana las asimetrías sociales, económicas, políticas, de poder y las condiciones institucionales que limitan la posibilidad de que el "otro" pueda ser considerado como un sujeto con identidad.

3. Agenciar espacios de encuentro, diálogo, respeto y asociación entre seres y saberes, sentidos y prácticas distintas, lo propio y lo ajeno, es decir, construir espacios posibilitadores de encuentros en y desde la diversidad.

4. Fomentar dentro de los espacios de encuentro intercultural la reflexión y la crítica con el fin de construir una nueva ciudadanía de niñas, niños, jóvenes y personas adultas que aprendan a vivir juntos en contextos cada vez más diversos.

\section{Resultados}

En este apartado tras la caracterización de la propia práctica pedagógica, y las consideraciones realizadas en los componentes conductuales de las representaciones docentes sobre lo juvenil, la diversidad, la cultura, la educación rural y la intercultural, se reconocen acercamientos a las nociones y principios de la interculturalidad como enfoque educativo dentro de las prácticas pedagógicas de las personas docentes participantes del estudio.

Las nociones y principios de la educación intercultural presentes en la práctica pedagógica se asocian principalmente a generar espacios para el diálogo, la escucha, experiencias de trabajo colaborativo, de debate de ideas y criticidad, motivación y reflexión hacia el logro de metas, la importancia del respeto. Todas las personas docentes consideran importante establecer una buena relación con sus 
estudiantes, tienen una disposición importante hacia las problemáticas juveniles, unos más que otros muestran apertura en conocer el mundo juvenil como es el caso de PMB3, PCT1, PMB4 y PP2, aspecto que les permite interactuar de mejor manera con las personas jóvenes.

La interculturalidad y las categorías que se han considerado en las entrevistas no se abordan en los establecimientos educativos como un proyecto o plan institucional dirigido a toda la comunidad educativa, según las docentes y los docentes este sería un paso importante en su abordaje, “...no se trabaja, no se incorpora fuertemente y los establecimientos quizás pondrían integrarlos con un programa interno que fuera paralelo a lo que el Ministerio lo presenta, pero tampoco se hace" (PB1), sino que de forma personal cada docente genera espacios dentro de sus prácticas pedagógicas para hacer lo que ellos consideran correcto y equitativo en el abordaje de la diversidad juvenil.

Dentro de las características docentes primordiales para atender la diversidad juvenil se encuentran el diálogo, la escucha, la comprensión y el fomentar vínculos con sus estudiantes, "otra cosa que yo creo que es importante, la capacidad de poder vincularme con el estudiante, la vinculación..." (PMP2) para poder comprender mejor a las personas jóvenes y sus distintas situaciones de vida.

La disposición tanto de PMP1 como de PMP2 a conocer el mundo en el que las personas juveniles viven y el reconocer en la interacción el mundo del otro, genera que en su práctica pedagógica se dé un aprendizaje en y desde la diversidad, esta diversidad es incorporada en la práctica pedagógica. Es muy importante señalar una cualidad docente que considera nociones de la educación intercultural como lo es la empatía, señala PMP1 "yo me admiro de cómo manejo la situación y tengo la competencia, que alguna vez un psicólogo lo dijo conversando conmigo, cuando yo estaba en la universidad me dijo 'tú tienes la capacidad de ponerte en el lugar del otro', entonces eso a mí me ha ayudado mucho, en entender la situación de la familia por ejemplo...".

Una noción y principio preponderante desde la mirada intercultural es la disposición hacia la diversidad, la sensibilidad y la apertura a conocer el mundo del otro, cualidad que se ve presente en PMP1, PP2, PCT1 y PMB4 de forma sobresaliente, "Conócelos, cuando uno se para frente a un curso son todos diferentes y uno tiene que mostrar su carácter... los dos cursos en los que trabajo no son nada de iguales en realidad, pero a los dos les gusta que uno converse con ellos..." (PMB4) agrega PMB3 la importancia de desarrollar relaciones constructivas entre adultos y jóvenes basadas en el respeto.

Por su parte PCT2 hace alusión a tres elementos propios de su práctica pedagógica, como importante de considerar en el trabajo con personas jóvenes el "saberlos entender", "el llegar a consensos con ellos", "no contestarles con agresividad tampoco", nociones que se consideran importantes desde una mirada intercultural, este último punto es coincidente con lo planteado por PB1 en torno ante situaciones difíciles, mantener la calma y luego hablar.

Otros aspectos importantes de seguir señalando son los planteados por PP2 docente con formación pedagógica que al igual que PMB3 son las que tienen mayor tiempo laborando en educación, ambas docentes se interesan por las problemáticas de sus estudiantes, PP2 enfatiza "Sí, creo que me caracterizo por eso, sí creo que de repente mucho", el mantener buenas relaciones entre docente-estudiante es un principio importante en la interacción pedagógica menciona la docente, y que ella considera en un porcentaje alto de educadores en el Liceo lo han logrado, señala de manera anecdótica la siguiente situación, "Si en un focus group, una niña de mi curso que asistió, decía que una de las cosas que más habían recalcado los chiquillos era la buena relación con los profesores, entonces yo siento que sí se sienten escuchados por nosotros...".

PMB3 es una profesora que dentro de su práctica pedagógica "tira a los estudiantes para arriba" los motiva a seguir adelante, una joven a la que ella ya no da clases, le decía, recuerda, "profe, se acuerda cuando nos dijo que nosotros éramos buenos, que podíamos, que valíamos. Bueno, eso es lo que digo yo, cada vez que estamos cayendo, digo vamos, sí podemos, nosotros podemos, y ella está en otro curso cachái, entonces pa' mí es una satisfacción".

Para PP1 al igual que PMB2 es importante el fomentar el trabajo en equipo, la integración grupal, el respecto, como señala específicamente, dentro de las clases de consejo de curso y de orientación PP1 aborda el trabajo en equipo, que el estudiantado se integren, "ellos tienen que integrarse, porque de repente te encuentras con cursos, cursos que están súper desunidos, o sea ahí hay un trabajo que 
tienes que hacer tú, en, en tu hora de consejo de curso, de, de hacer dinámica y que todos trabajen, de hablar con ellos, conversar...". Favorecer la unión del grupo, la convivencia estudiantil es una temática que tanto PP1, PP2, PCT1, PMP1, PMP2, PMB3, PMB4, PTT2 reconocen como necesaria y trabajan con sus cursos en las clases de orientación y consejo de curso. Argumenta PP1, que otra de las razones por las que aborda el tema del trabajo colaborativo es por el hecho de que el mercado laboral donde se van a insertar como técnicos en contabilidad es muy competitivo y enseñarles estas competencias es relevante.

Los debates en clase, espacios para que las personas jóvenes preparen su opinión con argumentos claros y definidos sobre un tema específico, es una práctica pedagógica importante y que caracteriza a PTT2, docente con formación técnica, la cual, la emplea para favorecer que el estudiantado se exprese de forma crítica y segura, asimismo fomenta que se respeten las opiniones aunque no concuerden con las propias.

Muy acorde al planteamiento anterior, PP2 emplea las películas en la sala de clase como estrategia pedagógica crítico-reflexiva, menciona PP2 "Sí, mira trabajamos hace poco ciudad de Dios en realidad nacional y los hice que buscaran críticas porque ellos tenían una postura, pero necesito que ellos, ellos entiendan que esa postura no es la única..."”

A modo de crítica y como un planteamiento interesante desde la mirada intercultural, PP2 señala no le gusta emplear la palabra tolerar porque siente que se refiere a aguantar al otro, cuando hablamos de incluir hay que tener cuidado de no acentuar diferencialismos al reconocer la diferencia, ya que al etiquetar otorgamos un valor social y a la vez "creamos una realidad que después se nos puede volver en contra", es importante señalar que actuamos a partir de eso que creamos afirma PP2. Con la docente se realiza una reflexión interesante que es importante de recalcar, y es el hecho de que en este camino reflexivo al que nos convoca la interculturalidad nos encontramos con más preguntas que respuestas, "Es como cuando abre, uno abre un link en una, en una página web y se te abren miles de cosas entonces es como una caja de Pandora, o sea, nunca acaba" (PP2).

El respeto es importante para todas las docentes y los docentes, sin embargo de forma puntual PTT2 expresa "Sí, o sea, a mí me gusta que los alumnos se respeten entre ellos. Así como yo los respeto, ellos me respeten...". Asimismo la interacción es un principio fundamental para la interculturalidad que tanto PB1, como PCT1, PP2, PP1, PMB3, PMB4, PTT2 señalan como característico de su práctica pedagógica, “por lo menos yo como docente me esfuerzo, en que eso funcione y si eso no funciona, la clase no te funciona, me entiende, porque tiene que haber diálogo con los alumnos, porque tiene que haber una escucha activa y participativa, pero es difícil, es muy difícil de conseguir, es muy difícil, los niños han cambiado, los chiquillos han cambiado mucho y es difícil" (PB1).

Prácticas pedagógicas que según $\mathrm{PCT} 1$ pueden favorecer el encuentro con el otro, y la interacción juvenil o lo que PB1 señala como "relaciones juveniles interculturales" consisten en enfocarse en hacer que las personas jóvenes se relacionen mediante el baile, las artes o el deporte, menciona "hacer algunas actividades como lo que estoy haciendo en la cueca, de que ya va en las dos pierde cueca y después cambio de pareja y el que queda solo paga alguna penitencia", otra práctica interesante en cuanto a fomentar la participación juvenil es el "cierre de la verdad, aquí van a mencionar todo lo que sienten...".

Se puede apreciar por parte de PCT1 una apertura hacia la comprensión de la diversidad, a favorecer el encuentro con el otro no solo desde el discurso, sino desde el generar como él lo denomina "redes de apoyo", "veo que mi curso está muy, está muy separado, estoy haciendo cosas para que se junten, actividades para que ellos se junten, con solo mencionarte a, hasta que les cuesta conversar con los representantes y directivos del curso" ante este panorama entre las actividades propuestas fueron "Yo en mi curso de jefatura hice una convivencia antes de finalizar el tema de las operaciones de invierno, hice una hamburguesa como los gringos y fue, y capté lo mismo había cinco niños acá, niñas acá y así y así y en el único momento que se juntaron fue cuando comieron, pero no para compartir, un antes, eso yo no sé por qué está pasando acá”.

Las consideraciones anteriores permiten constatar que en las prácticas pedagógicas de docentes que laboran en contextos de interacción juvenil rural se pueden identificar nociones y principios de la educación intercultural importante de ser potencializados y empleados en el fortalecimiento 
de estrategias que permitan comprender y abordar la diversidad sociocultural de las personas jóvenes en las aulas rurales de establecimientos educativos de la educación media en Chile, desde una mirada intercultural. Sin embargo es relevante señalar siguiendo a autores como Aguado (2003) y Leiva (2011) que estas nociones y principios aún se encuentran en un plano más teórico que práctico, como se evidenció entre otros motivos por la carencia a nivel de formación inicial docente y de perfeccionamiento docente de la debida capacitación en educación intercultural.

\section{Consideraciones finales}

La nociones y principios del enfoque intercultural identificadas en las prácticas pedagógicas docentes, tanto en lo teórico asociado a los componentes reconocimiento y valoración y a lo práctico vinculado a la dimensión incorporación, son consideradas en un primer momento fortalezas del profesorado en la educación rural; en segundo lugar estos principios se constituyen en indicadores relevantes para agenciar procesos de capacitación y/o perfeccionamientos docente, así como insumos claros hacia dónde dirigir la formación inicial del profesorado, mostrando además un itinerario amplio sobre el cual estructurar políticas públicas en temas como diversidad, juventud rural, interculturalidad, ruralidad y cultura rural, en tanto como se identificó, uno de los aspectos en perspectiva intercultural que requiere de un mayor reforzamiento en la formación profesional docente, es favorecer el desarrollo de un pensamiento crítico que responda a las demandas de sociedades cada vez más pluriculturales.

Por otra parte, es preponderante señalar tras las consideraciones teóricas que sustentaron la investigación en contraste con los resultados obtenidos, lo emergente que resulta el enfoque intercultural en los contextos educativos estudiados, ya que si bien es cierto se pueden visualizar en las prácticas pedagógicas de docentes que se desempeñan en contextos de interacción juvenil rural, acercamientos a las nociones y principios de la educación intercultural, que permiten generar condiciones para el reconocimiento, valoración e incorporación de la diversidad y la cultural rural dentro de los establecimientos educativos de manera relevante, no se puede hablar aun de prácticas pedagógicas interculturales o de la presencia de un enfoque intercultural dentro de las prácticas pedagógicas docentes en sentido estricto.

La consideración anterior se fundamenta, en tanto aún hace falta que las prácticas pedagógicas realizadas en contextos de interacción juvenil rural, se ejecuten desde un acto consciente en perspectiva intercultural, que involucre trabajo reflexivo docente en el replanteamiento de la interacción con el otro, como un hecho vivencial y no solo teórico discursivo. Acción que considerando a Rehaag (2010) implica reconocer en la interacción, el mundo del otro desde la magnitud de lo que implica incluir al otro, es decir una puesta en práctica solidaria con lo propio y lo ajeno.

Estas consideraciones siguiendo a Leiva (2011) hacen que lo intercultural como enfoque emergente aun permanezca entre el deseo de ser una propuesta educativa crítica y la realidad de encontrarse entre la exaltación folclórica y un enfoque curricular compensatorio, pese a compartir este planteamiento con el autor, es importante considerar la necesidad de dinamizar y ampliar el constructo intercultural, en el que tenga cabida la diversidad no solo desde el otro diverso inmigrante o indígena sino el otro diverso por su condición de ser humano, una diversidad amplia como lo supone lo juvenil en la que tiene cabida, tanto lo que construye la persona joven desde sí misma y desde las construcciones que los demás realizan sobre ella, es decir una construcción intersubjetiva, que brinde la posibilidad de identificación con el otro sin dejar de ser uno mismo.

Los planteamientos señalados, recalcan que la heterogeneidad es la norma en espacios educativo rurales, no una excepción, así mismos se reitera la función hermenéutica que cumple la educación intercultural (Abdallah-Pretceille, 2006) ya que permite agenciar mecanismo teóricos y prácticos para la (re) comprensión de las prácticas pedagógicas valorando la multiplicidad de referentes que la complejizan, y la condiciones que posibilitan que el profesorado dé un salto a la realización de prácticas pedagógicas interculturales ${ }^{1}$. 


\section{Referencias Citadas}

Abdallah-Pretceille, $\mathrm{M}$.

2006 El paradigma intercultural como mirada hacia la diversidad. Actas Congreso INTER. Madrid. Servicio Publicaciones UNED. Intercultural Recuperado de http:// www.uned.es/congreso-inter-educacion-intercultural.

Aguado, T.; Ballesteros, B.; Malik, B.; Sánchez, M.

2003 Educación intercultural en la enseñanza obligatoria: prácticas escolares; actitudes y opiniones de padres, alumnos y profesores; resultados académicos de los estudiantes de diversos grupos culturales, en Revista de Investigación Educativa (RIE), 21(2), 323-348. ISSN: 0212-4068. - See more at: http://www.uned.es/grupointer/articulos.html\#sthash. vTNUGtiH.dpuf

Aguado, T.

2011 El enfoque intercultural en la búsqueda de buenas prácticas escolares. Revista Latinoamericana de Inclusión Educativa, 5(2), pp. 23-42. ISSN: 0718-7378 (edición electrónica), ISSN: 0718-5480 - See more at: http://www. uned.es/grupointer/articulos.html\#sthash.vTNUGtiH. dpuf

2005 Educación Intercultural: Una propuesta para la transformación de la escuela. Ministerio de Educación y ciencia. Madrid, España.

2003 Pedagogía intercultural. Madrid: Mc.Graw-Hill.

1995 Un modelo de Orientación para la comprensión y competencia multiculturales. Revista Española de Orientación y Psicopedagogía, ISSN 1139-7853, ISSN-e 1989-7448, Vol. 6, No 10, 1995, pp. 105-111.

Aguado, T.; Gil, I.; Mata, P.

2008 El enfoque intercultural en la formación del profesorado. Dilemas y propuestas. Universidad Nacional de Educación a Distancia (UNED), Madrid, España.

2005 Educación Intercultural: Una propuesta para la transformación de la escuela. Ministerio de Educación y ciencia. Madrid, España.

Aguado, T.; Herraz, M.

2006 Medición social intercultural en el ámbito socioeducativo. Universidad Nacional de Educación a Distancia (UNED). Madrid, España.

Bárcena, F.

2000 La práctica reflexiva en educación. Editorial Complutense, Madrid. España.

Betancourt, R.

2004 Reflexiones de Raúl Betancourt sobre el concepto de Educación Intercultural, México, DF.

Bueno-Abad, J.

2000 Concepto de representaciones sociales y exclusión. Facultad De Ciencias Sociales Universidad de Valencia, España.

Cabrera, R.

2009 Prácticas Pedagógicas para una Convivencia Intraescolar Democrática. (Tesis de Maestría, Universidad Andina Simón Bolívar, Ecuador). Recuperado de http://repositorio.uasb. edu.ec/handle/10644/966

Cáceres, $\mathrm{P}$.

2003 Análisis Cualitativo de Contenido: Una alternativa metodológica alcanzable. Revista Psicoperspectivas de la escuela de psicología. Facultad de filosofía y educación pontificia universidad católica de Valparaíso. vol. II / 2003 (pp. 53-82).

Canales, $\mathrm{M}$.

2006 Metodologías de Investigación Social. Introducción a los oficios. LOM Ediciones, Santiago, Chile.

Carr, W.

2002 Una Teoría para la Educación. Hacia una investigación educativa critica. Ediciones Morata, S.L. Tercera Edición. Madrid, España.

Delgado, J. M.; Gutiérrez, J.

2007 Métodos y técnicas cualitativas de investigación en ciencias sociales. Madrid: Síntesis.

Dietz, G.

2003 Multiculturalismo, interculturalidad y educación. Una aproximación antropológica. Editorial Universidad de Granada, España

2012 La Educación Intercultural hoy; Debates teóricos y prácticos. Conferencia presentada en la Universidad de Santiago de Chile, celebrado en Santiago, Chile, el 9 de noviembre de 2012.

Duschatzky, S.; Skliar, C.

2000 La diversidad bajo sospecha. Reflexiones sobre los discursos de la diversidad y sus implicaciones educativas. Cuadernos de pedagogía Rosario $N^{\circ} 7$. Buenos Aires Argentina.

Duzchatzky, S.; Sztulwark, D.

2011 Imágenes de lo no escolar, en la Escuela y más allá. Paidós, Tramas sociales 66. Buenos Aires, Argentina.

Erazo, S.

2012 Metodologías de investigación Cualitativa. (Material mimeográfico del curso). Universidad de Santiago de Chile. Programa de Doctorado en Educación, Mención en Educación Intercultural.

2011 Prácticas reflexivas, racionalidad y estructura en contextos de interacción profesional. IISUE-UNAM, perfiles educativos, Vol. XXXIII, No 133.

2001 Innovación de las prácticas de planificación curricular en la escuela y en el liceo: Una Estrategia para la Apropiación, Contextualización y Complementación de los Planes y Programas propuestos por el Mineduc. Pensamiento Educativo. Vol. 29, pp. 245-275.

Escarbajal, A.

2010 Interculturalidad, Medición y trabajo colaborativo. NARCEA, S.A. Ediciones Madrid, España.

Galindo, J.

2009 Comunicología, etnometodología y comunicometodología: la comunicación como acción y como representación reflexiva constructiva. Razón y palabra, ISSN-e 1605-4806, $\mathrm{N}^{\circ} 67$.

Jodelet. D.

1986 La representación social: fenómenos, conceptos y teoría. En Moscovici, S. Psicología social II. Pensamiento y vida social. Psicología social y problemas sociales. BarcelonaBuenos Aires-México: Paidós.

Jurado, C.; Tobasura, I.

2012 Dilema de la juventud en territorios rurales de Colombia: ¿campo o ciudad? Revista Latinoamericana de Ciencias Sociales, Niñez y Juventud, 10 (1), pp. 63-77. 
Recuperado de http://revistaumanizales.cinde.org.co/index. php/revistalatinoamericana/article/view/581/314

Kessler, G.

2005 Estado del Arte de la Investigación Sobre Juventud Rural en América Latina.

Leiva, O. J.

2011 La Educación Intercultural en una encrucijada de caminos: reflexiones pedagógicas para la construcción de una escuela intercultural. Vol. 4. Núm. 7. Pp. 43-56. Revista digital del centro del profesorado Cuevas-Olula (Alemeria). Espiral. Cuadernos del profesorado.

Merchán, Mora, J.

2001 La producción del conocimiento escolar en la clase de historia; profesores, alumnos y prácticas pedagógicas en la Educación Secundaria. (Tesis Doctoral, Universidad de Sevilla). Recuperado de: www.ceuandalucia.com/programas/ CursosAnteriores/Programas 10-11.pdf

Perrenound, P.

2007 Desarrollar la práctica reflexiva en el oficio de enseñar. Personalización y razón pedagógica. Colección Crítica y fundamentos. Editorial, GRAÓ. Barcelona. España.

Rehaag, I.

2010 La perspectiva Intercultural en la Educación. El Cotidiano, Núm.- 160. Pp.75-83. Universidad Autónoma Metropolitana Unidad Azcapotzalco, DF, México. Recuperado de: http:// www.redalyc.org/articulo.oa?id=325127660009

Rodríguez, $\mathrm{N}$.

2007 "Prácticas docentes y mejora de la escuela", Educere, vol. 11, núm. 39, pp. 699-708.

Sagastizabal, M.; Perlo, C.; Pivetta, B.; San Martín, P.

2009 Aprender y enseñar en contextos complejos. Multiculturalidad, diversidad y fragmentación. Centro de Publicaciones Educativas y material didáctico, Buenos Aires, Argentina.
Suárez, M.

2001 Teoría pedagógica, necesidades e impacto en el quehacer docente. Una aproximación a la formación docente en Venezuela, desde el espacio universitario. Universidad Rovira, Tarragona, España.

Torres, C.

2001 Democracia, educación y multiculturalismo: dilemas de la ciudadanía en un mundo global (México D.F.: Siglo XXI). Capítulos 6 y 7.

Vasilachis de Gialdino, I.

2009 Los fundamentos ontológicos y epistemológicos de la investigación cualitativa. Buenos Aires, Argentina

Velazco, A.

2007 Un sistema para el análisis de la interacción en el aula. Revista Iberoamericana de Educación. Venezuela.

Villalta, M.

2009 Análisis de la conversación. Una propuesta para el estudio de la Interacción Didáctica en Sala de Clase. Revista Iberoamericana de Educación, Estudios Pedagógicos XXXV, $\mathrm{N}^{\circ} 1$.

Walsh, C.

2005 La Interculturalidad en la Educación. Ministerio de Educación. Dirección Nacional de Educación Bilingüe Intercultural (DINEBI). Lima-Perú.

Williamson, G.

2008 Estudio sobre la educación para la población rural en Chile. Educación para la Población Rural en Brasil, Chile, Colombia, Honduras, México, Paraguay y Perú. Proyecto FAO-UNESCODGCS/ITALIA-CIDE-REDUC. Recuperado de http://unesdoc. unesco.org/images/0013/001360/136025m.pdf

Zemelman, $\mathrm{H}$.

1992 Los horizontes de la razón I, uso crítico de la teoría; Dialéctica y apropiación de presente. Editorial del hombre, México.

\section{Nota}

1 Resultados Investigación Doctoral, Universidad de Santiago de Chile, Programa Ciencias de la Educación, mención en Educación Intercultural, período 2012-2015. 